\title{
THERMOMECHANICS AND THE FORMULATION \\ OF THE STEFAN PROBLEM \\ FOR FULLY FACETED INTERFACES
}

BY

MORTON E. GURTIN (Carnegie Mellon University, Pittsburgh, PA)

AND

JOSÉ MATIAS (Istituto Superior Tecnico, Lisbon, Portugal)

\begin{abstract}
This paper develops a thermomechanics of two-phase heat conductors in which the interface between phases is fully faceted. The theory is based on balance of forces, balance of energy, and growth of entropy in conjunction with constitutive equations for the interface; and the chief result is a free-boundary problem of Stefan type in which the classical interface condition $u=0$ is replaced by a condition relating the integral of $u$ over each facet to the normal velocity of that facet.
\end{abstract}

Introduction. The classical theory of solidification is based on a free-boundary problem consisting of the bulk energy balance

$$
c_{\mu} \dot{u}=-\operatorname{div} \mathbf{q}, \quad \mathbf{q}=-\mathbf{K}_{\mu} \nabla u
$$

in each phase $\mu=\alpha, \beta$, balance of energy

$$
\ell V=[\mathbf{q}] \cdot \mathbf{m}
$$

across the interface $\mathscr{S}=\mathscr{S}(t)$, and the Stefan condition

$$
u=0
$$

on $\mathscr{S}$, supplemented by suitable initial and boundary conditions. Here

$$
u=\left(\vartheta-\vartheta_{T}\right) / \vartheta_{T}
$$

with $\vartheta$ the temperature and $\vartheta_{T}$, a material constant, the transition temperature; [q] is the jump in the heat flux $\mathbf{q}$ across $\mathscr{S} ; \ell$ is the latent heat; $c_{\mu}$ and $\mathbf{K}_{\mu}$ are the specific heat and conductivity tensor (multiplied by $\vartheta_{T}$ ); $\mathbf{m}$ is the orientation (the unit normal to $\mathscr{S}$ directed outward from $\alpha) ; V$ is the normal speed ${ }^{1}$ of $\mathscr{S}$.

Received May 20, 1993.

1991 Mathematics Subject Classification. Primary 80A22; Secondary 73B30.

${ }^{1}$ We use the term speed even though we do not restrict $V$ to be positive. 
In the presence of interfacial energy and transformation kinetics the Stefan condition (1.3) is replaced by a condition ${ }^{2}$

$$
\ell u=\left\{f(\mathbf{m}) \mathbf{1}+D^{2} f(\mathbf{m})\right\} \cdot \mathbf{L}-b(\mathbf{m}) V,
$$

where $\mathbf{L}$ is the curvature tensor of $\mathscr{S}, f(\mathbf{m})$ is the interfacial free energy and $b(\mathbf{m})$ the kinetic modulus at $\vartheta_{T}$, and $D^{2} f(\mathbf{m})$ is the second derivative of $f(\mathbf{m})$ on the unit sphere.

Many materials are characterized by interfacial energies, termed crystalline, for which $f(\mathbf{m})$ has cusps at orientations $\mathbf{m} \in \mathscr{M}$, with $\mathscr{M}$ a finite set representing the low-energy orientations of the interface. ${ }^{3}$ Since $D^{2} f(\mathbf{m})$ is "infinite" at $\mathbf{m} \in \mathscr{M}$, it seems reasonable to expect, as a formal consequence of (1.5), that the interface is flat $(\mathbf{L}=\mathbf{0})$ for orientations $\mathbf{m} \in \mathscr{M}$, a conclusion reached by Herring, ${ }^{4}$ who proposed the interface condition

$$
\ell \int_{F} u(\mathbf{x}, t) d A(\mathbf{x})=\sum_{G} \lambda_{F G} L_{F G}(t)-A_{F}(t) b\left(\mathbf{m}_{F}\right) V_{F}(t)
$$

on each facet $F=F(t)$, where $\mathbf{m}_{F}, V_{F}(t)$, and $A_{F}(t)$ are the orientation, normal speed, and area of $F$; the sum is over all facets $G$ adjacent to $F ; L_{F G}(t)$ is the length of the edge $F \cap G$; and

$$
\lambda_{F G}=\left\{\left(\mathbf{m}_{F} \cdot \mathbf{m}_{G}\right) f\left(\mathbf{m}_{F}\right)-f\left(\mathbf{m}_{G}\right)\right\} /\left\{1-\left(\mathbf{m}_{F} \cdot \mathbf{m}_{G}\right)^{2}\right\}^{1 / 2} .
$$

Here it is tacit that the orientations of $\mathscr{S}$ are confined to $\mathscr{M}$, so that $\mathscr{S}$ is fully faceted. Interestingly, in contrast to the classical Stefan condition $u=0,(1.6)$ represents a condition on the integral of $u$ over each facet $F$, and is hence nonlocal.

It is the purpose of this paper to develop a thermomechanics of two-phase heat conductors in which the interface between phases is fully faceted, with orientations m confined to a finite set $\mathscr{M}$. Following Gurtin [1988], we base the theory on balance of forces, balance of energy, and growth of entropy in conjunction with constitutive equations for the interface giving the free energy $f$, the entropy $s$, and the normal interaction $\pi$ (the normal force exerted by the bulk material on the interface) in terms of $\theta, \mathbf{m} \in \mathscr{M}$, and $V$. Using the Coleman-Noll procedure $[1963]^{5}$ to restrict these constitutive equations, we find that (i) the free energy and entropy are independent of $V$ and related in the classical manner; (ii) the normal interaction has the form

$$
\pi=[\psi]-\hat{b}(\vartheta, \mathbf{m}, V) V
$$

with $\psi$ the bulk free energy and $\hat{b}(\vartheta, \mathbf{m}, V) \geq 0$ a kinetic modulus.

\footnotetext{
${ }^{2} \mathrm{Cf}$. Gurtin [1988, 1993a] for references to the extensive literature on free-boundary conditions of this type and for a derivation of (1.5) within the framework of continuum thermodynamics.

${ }^{3} \mathscr{M}$ is the exact set of orientations that appear on the Wulff shape (the unique crystal shape that minimizes total interfacial energy at fixed volume).

${ }^{4}$ [1951], Eq. 15, for $V=0$. An equivalent version of (1.6), for the interface a polygonal curve in the plane, was proposed somewhat later, but independently, by Ben Amar and Pomeau [1988] and by Gurtin and Matias [1990].

${ }^{5}$ As generalized by Gurtin [1988] to two-phase materials.
} 
The free-boundary conditions resulting from these general constitutive equations and balance laws are complicated, and for that reason we consider a simplified theory in which the interface conditions are linearized in the variables $u$ and $V$; the resulting system, which we refer to as the quasi-linear system, consists of the bulk equations (1.1) supplemented by the interface conditions (1.2) and (1.6).

The quasi-linear system is an approximation of the general theory and cannot be expected to obey the general laws of energy balance and entropy growth. We modify the quasi-linear system by adding "higher-order" terms which give the theory an approximate thermodynamic structure; when $c_{\alpha}=c_{\beta}$-a condition trivially satisfied in the quasi-static theory obtained by setting $c_{\alpha}=c_{\beta}=0$-the modified system reduces to the quasi-linear system.

Global growth conditions are established for the modified system in a bounded domain under various boundary conditions. In particular, for quasi-static situations with the boundary insulated and disjoint from the interface, the total interfacial free energy (at the transition temperature) decreases, while the volume of each phase remains constant.

We next consider situations in which the conductivities of the individual phases are small and the boundary is held at the constant temperature $U$, and give a plausibility argument leading to the evolution equation ${ }^{6}$

$$
b\left(\mathbf{m}_{F}\right) V_{F}(t)=A_{F}(t)^{-1} \sum_{G} \lambda_{F G} L_{F G}(t)-D
$$

for the interface, with $D$ a constant that depends only on $U$.

We introduce a notion of admissibility for the interface which requires that (i) orientations of adjacent facets be adjacent orientations for the Wulff shape; (ii) the complete set of orientations of facets meeting in a corner must be a complete set also for the Wulff shape. Granted admissibility, we establish a simple formula for the $\lambda$ 's of (1.7) in terms of the gradient of the interfacial energy, extended in a convex manner from $\mathscr{M}$ to all of $\mathbb{R}^{3}$.

\section{Crystalline bodies. Kinematics.}

a. Crystalline bodies. We consider a body consisting of two phases, $\alpha$ and $\beta$, separated, at each time $t$, by a fully faceted interface $\mathscr{S}(t)$, and write $\Omega_{\alpha}(t)$ and $\Omega_{\beta}(t)$ for the subregions of the body occupied by $\alpha$ and $\beta$. We assume that the body occupies all of $\mathbb{R}^{3}$, and that $\Omega_{\alpha}(t)$ and $\Omega_{\beta}(t)$ are closed polygonal regions with $\mathbb{R}^{3}$ as their union and $\mathscr{S}(t)$ as their intersection. Then $\mathscr{S}(t)$ is the union of a finite number of closed flat sides $F(t)$, its facets; adjacent facets $F(t)$ and $G(t)$ intersect along line segments $F(t) \cap G(t)$, its edges; and edges intersect in corners. We orient $\mathscr{S}(t)$ by a choice of unit normal field $\mathbf{m}(\mathbf{x}, t)$, the orientation of $\mathscr{S}(t)$, chosen so that

$$
\mathbf{m}(\mathbf{x}, t) \text { coincides with the outward unit normal to } \partial \Omega_{c(}(t) \text {. }
$$

We assume that each facet $F(t)$ has orientation $\mathbf{m}_{F}$ independent of $t$, and that the position vector of each corner varies smoothly in time. (A tacit assumption is that

\footnotetext{
${ }^{6}$ Proposed by Taylor [1988], Angenent and Gurtin [1989], and Giga, Gurtin, and Matias [1993].
} 
facets are neither created nor destroyed.) We denote by $V(\mathbf{x}, t)$ the normal speed of $\mathscr{S}(t)$ in the direction $\mathbf{m}(\mathbf{x}, t)$; since the facet normals are constant, each facet $F(t)$ has normal speed $V_{F}(t)$ a function only of $t$.

b. Bulk and interfacial fields. Our theory is characterized by (1) bulk fields defined in $\Omega_{\alpha}(t)$ and $\Omega_{\beta}(t)$ for all $t$ and allowed to suffer jump discontinuities across the interface away from its edges; and (2) interfacial fields defined on $\mathscr{S}(t)$ for all $t$, and allowed to suffer jump discontinuities across edges. No restrictions are placed on the (possibly quite singular) behavior of bulk fields at edges. For $\Phi$ a bulk field, we write $\Phi_{\alpha}$ and $\Phi_{\beta}$, respectively, for the limits of $\Phi$ as the interface is approached from the $\alpha$ and $\beta$ regions, and [ $\Phi]$ for the jump in $\Phi$ across the interface:

$$
[\Phi]=\Phi_{\beta}-\Phi_{\alpha} .
$$

Similarly, given an interfacial field $\varphi$ and a facet $F$, we write $\varphi_{F}$ for the limit of $\varphi$ as $\partial F$ is approached from $F$ :

$$
\varphi_{F}(\mathbf{x}, t)=\lim _{\substack{\mathbf{y} \rightarrow \mathbf{x} \\ \mathbf{y} \in F(t)}} \varphi(\mathbf{y}, t)
$$

We use the term tensor for linear transformation of vectors (elements of $\mathbb{R}^{3}$ ) into vectors: $\mathbf{1}$ is the identity tensor, $\mathbf{C}^{\mathbf{T}}$ is the transpose of a tensor $\mathbf{C}$, and $\mathbf{a} \otimes \mathbf{b}$ is the tensor product of vectors $\mathbf{a}$ and $\mathbf{b}$. Further, we write

$$
\mathbf{P}=\mathbf{1}-\mathbf{m} \otimes \mathbf{m}
$$

$\mathbf{P}(\mathbf{x}, t)$ is the projection onto the tangent plane for $\mathscr{S}(t)$ at $\mathbf{x}$.

Given an interfacial scalar field $\varphi$, we denote by $\stackrel{\circ}{\varphi}$ the normal time-derivative ${ }^{7}$ of $\varphi$ (the derivative following the normal trajectories of $\mathscr{S}(t)$ ).

c. Control volumes. Velocities. Let $R$ be a control volume; that is, a bounded region $R \subset \mathbb{R}^{3}$ with piecewise-smooth boundary. We will consistently write

$$
\mu(t)=R \cap \mathscr{S}(t), \quad R_{r r}(t)=R \cap \Omega_{r r}(t), \quad R_{\beta}(t)=R \cap \Omega_{\beta}(t)
$$

for the intersection of $R$ with the interface and the $\alpha$ and $\beta$ phases. Then $r(t)$, when of nonzero area, has a piecewise-smooth boundary curve $\partial r(t)$ with welldefined outward unit normal $\nu_{\nu}(\mathbf{x}, t)$ tangential to $\mathscr{S}(t)$. We will refer to $R$ as a facet control volume at $t_{0}$ if $r(t)$ is contained in a single facet for all $t$ near $t_{0}$; we will refer to $R$ as an edge control volume at $t_{0}$ if, for all $t$ near $t_{0}, r(t)$ intersects exactly one edge and no corners.

Given a local parametrization $\mathbf{x}=\mathbf{r}(u, t)$ for $\partial \mu(t), \mathbf{w}(\mathbf{x}, t)=\partial \mathbf{r}(u, t) / \partial t$ satisfies $\mathbf{w} \cdot \mathbf{m}=V, \mathbf{w} \cdot \nu_{\mu}=W_{\nu}$, where $W_{\nu}$, the tangential speed ${ }^{8}$ of $\partial \mu(t)$, is independent

\footnotetext{
${ }^{7}$ Cf. Gurtin and Struthers [1990].

${ }^{8} \mathrm{~W}$, is actually the normal speed of the curve $\partial r$ in the tangent plane of $\mathscr{S}$; we use the term tangential to emphasize this latter property and to differentiate $W$, from the normal speed $V$ of $\mathscr{S}$.
} 
of the choice of parametrization. The motion of $\partial \mu(t)$ may be characterized intrinsically by the velocity field

$$
\mathbf{w}_{\nu}=V \mathbf{m}+W_{\nu} \nu_{\nu} .
$$

In terms of this notation, $W_{F}$ represents the tangential speed and $\mathbf{w}_{F}$ the intrinsic velocity for the boundary curve of a facet $F$; for $F$ and $G$ adjacent facets,

$$
\mathbf{w}_{F}=\mathbf{w}_{G}
$$

on $F \cap G$.

The following transport identities will be useful: for $\Phi$ a bulk scalar field,

$$
\left\{\int_{R} \Phi\right\}^{\cdot}=-\int_{r}[\Phi] V+\int_{R_{n}} \dot{\Phi}+\int_{R_{\beta}} \dot{\Phi}
$$

for $\varphi$ an interfacial scalar field and $R$ a facet control volume,

$$
\left\{\int_{\nu} \varphi\right\}^{\cdot}=\int_{\nu} \stackrel{\circ}{\varphi}+\int_{\partial \nu} \varphi W_{\nu} .
$$

3. Basic fields. The thermodynamics of the body is described by:

bulk fields

$$
\begin{array}{ll}
\varepsilon(\mathbf{x}, t) & \text { bulk internal energy (volume), } \\
\eta(\mathbf{x}, t) & \text { bulk entropy (volume), } \\
\vartheta(\mathbf{x}, t) & \text { absolute temperature, } \\
\mathbf{q}(\mathbf{x}, t) & \text { heat flux (area), }
\end{array}
$$

interfacial fields

$$
\begin{array}{ll}
e(\mathbf{x}, t) & \text { interfacial internal energy (area), } \\
s(\mathbf{x}, t) & \text { interfacial entropy (area), } \\
\mathbf{C}(\mathbf{x}, t) & \text { capillary stress (length), } \\
\boldsymbol{\pi}(\mathbf{x}, t) & \text { interaction force (area), } \\
h(\mathbf{x}, t) & \text { apparent heating (length), }
\end{array}
$$

with "(volume)" shorthand for "per unit volume", and so forth. Here $\varepsilon, \eta$, and $\vartheta$ are bulk scalar fields; $\mathbf{q}$ is a bulk vector field; $e, s$, and $h$ are interfacial scalar fields; $\mathbf{C}$ is an interfacial tensor field; $\boldsymbol{\pi}$ is an interfacial vector field.

We assume that the

$$
\text { temperature } \vartheta \text { is continuous across the interface; }
$$

generally, we will not specify regularity hypotheses other than to note that the remaining bulk fields are allowed to suffer jump discontinuities across the interface away from the edges and to exhibit singular behavior at the edges, and the interfacial fields are allowed to suffer jump discontinuities across the edges.

It is convenient to define the bulk and interfacial free energies through

$$
\psi=\varepsilon-\vartheta \eta, \quad f=e-\vartheta s .
$$


4. Balance of forces. We assume that the force balance ${ }^{9}$

$$
\int_{\partial r} \mathbf{C} \boldsymbol{\nu}+\int_{r} \boldsymbol{\pi}=\mathbf{0}
$$

is satisfied for all control volumes $R$ that intersect the interface, where $r(t)=R \cap$ $\mathscr{S}(t)$ and $\boldsymbol{\nu}=\nu_{\mu}$. The first integral gives the force on $r(t)$ exerted across $\partial r(t)$ by the portion of $\mathscr{S}(t)$ exterior to $\mu(t)$; the second integral gives the force exerted on $r(t)$ by the portions of the bulk material adjacent to the interface. Although $\mathbf{C v}$ is defined on each vector $\mathbf{v}$, it is clear from (4.1) that its action on vectors normal to $\mathscr{S}(t)$ is irrelevant, and for that reason we add the restriction

$$
\mathbf{C m}=\mathbf{0} \text {. }
$$

Let $F$ and $G$ be adjacent facets. Then the force balance (4.1), applied to an edge control volume for $F \cap G$, yields, upon shrinking $r$ to $F \cap G$, the edge balance

$$
\mathbf{C}_{F} \boldsymbol{\nu}_{F}=-\mathbf{C}_{G} \boldsymbol{\nu}_{G}
$$

on $F \cap G$, where we have used the notation (2.3), and where $\nu_{F}$ and $\nu_{G}$ are the outward unit normals to the boundary curves $\partial F$ and $\partial G$.

Given any control volume $R$, the rate at which the capillary stress does work on $R$ is assumed given by ${ }^{10}$

$$
\int_{\partial r} \mathbf{C} \nu \cdot \mathbf{w}_{\text {, }}
$$

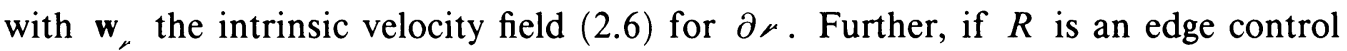
volume for $F \cap G$, then, writing

$$
\mu_{F}(t)=\mu(t) \cap F(t), \quad r_{G}(t)=\mu(t) \cap G(t),
$$

we may use (2.7) and (4.3) to conclude that

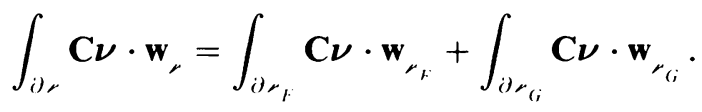

We assume that the capillary stress $\mathbf{C}$ has the form

$$
\mathbf{C}=\sigma \mathbf{P}+\mathbf{m} \otimes \mathbf{c}
$$

with $\sigma$ a scalar field and

$$
\mathbf{c}=\mathbf{C}^{\mathrm{T}} \mathbf{m}
$$

a tangential vector field; the action $\mathbf{C} \boldsymbol{\nu}$ of $\mathbf{C}$ on a tangential field $\boldsymbol{\nu}$ then consists of a component $\sigma \nu$ tangent to $\mathscr{S}$ and a component $(\mathbf{c} \cdot \boldsymbol{\nu}) \mathbf{m}$ normal to $\mathscr{S}$, so that $\sigma$ represents the surface tension and $\mathrm{c}$ the surface shear. Using (4.7), we can rewrite (4.4) as

$$
\int_{i, r} \mathbf{C} \boldsymbol{\nu} \cdot \mathbf{w}_{,}=\int_{i \nu}\left\{\sigma W_{\nu}+V \mathbf{c} \cdot \boldsymbol{\nu}\right\}
$$

REMARK. The specific form (4.7) for $\mathbf{C}$ follows from an argument of Gurtin and Struthers [1990]. The intrinsic velocity field $\mathbf{w}_{\mu}$ in (4.4) is replaced by a velocity

\footnotetext{
${ }^{9}$ Gurtin [1988].

${ }^{10}$ Gurtin and Struthers [1990]. Gurtin [1991].
} 
field $\mathbf{w}$ computed using an arbitrary local parametrization $\mathbf{x}=\mathbf{r}(u, t)$ for $\partial \mu(t)$. The requirement that (4.4) be independent of the specific choice of parametrization then yields-by virtue of (iii) of the Invariance Lemma of Gurtin and Struthers [1990]-the representation (4.7).

By (4.7), the balance (4.3) is equivalent to the relation

$$
\sigma_{F} \boldsymbol{\nu}_{F}+\left(\mathbf{c}_{F} \cdot \boldsymbol{\nu}_{F}\right) \mathbf{m}_{F}=-\left[\sigma_{G} \boldsymbol{\nu}_{G}+\left(\mathbf{c}_{G} \cdot \boldsymbol{\nu}_{G}\right) \mathbf{m}_{G}\right] \text {, }
$$

which may be solved (uniquely) for $\mathbf{c}_{F} \cdot \nu_{F}$ and $\mathbf{c}_{G} \cdot \nu_{G}$; in particular,

$$
\mathbf{c}_{F} \cdot \boldsymbol{\nu}_{F}=\left[\left(\mathbf{m}_{F} \cdot \mathbf{m}_{G}\right) \sigma_{F}-\sigma_{G}\right] /\left[1-\left(\mathbf{m}_{F} \cdot \mathbf{m}_{G}\right)^{2}\right]^{1 / 2}=: \Lambda_{F G} .
$$

Consider next (4.1) applied to the facet $F$. If we take the inner product of (4.1) with (the constant vector) $\mathbf{m}_{F}$, we find that

$$
\int_{\partial F} \Lambda_{F G}=-\int_{F} \pi
$$

where

$$
\pi=\pi \cdot \mathbf{m}
$$

is the normal interactive force.

Since $c$ is a tangential vector field, its surface divergence on each facet may be formally identified with its ordinary divergence div c, and, in view of (4.7), the same applies to the divergence $\mathbf{C}$. Similarly, we may formally identify the surface gradient of $\sigma$ with $\mathbf{P} \nabla \sigma$. Thus

$$
\operatorname{div} \mathbf{C}=\mathbf{P} \nabla \sigma+\mathbf{m} \operatorname{div} \mathbf{c} .
$$

The balance (4.1) therefore has the local form

$$
\operatorname{div} \mathbf{C}+\boldsymbol{\pi}=\mathbf{0}
$$

on each facet, or equivalently

$$
\operatorname{divc}+\pi=0, \quad \mathbf{P} \nabla \sigma+\mathbf{P} \boldsymbol{\pi}=\mathbf{0}
$$

on each facet.

REMARKS.

1. The intrinsic motion of the interface is normal; tangential motion is irrelevant. For that reason, we will not specify the tangential component $\mathbf{P} \boldsymbol{\pi}$ of $\boldsymbol{\pi}$ constitutively, but instead will consider $\mathbf{P} \boldsymbol{\pi}$ as determined by $(4.16)_{2}$.

2. Given the interfacial fields $\sigma$ and $\pi$, let $\Lambda_{F G}$ be defined by (4.11), and suppose that (4.12) is satisfied. Let $\mathrm{c}$ be a tangential field defined on each facet $F$ as the solution of the boundary-value problem consisting of (4.11) and (4.16) $)_{1}$. (Such a solution exists because of (4.12).) Then, defining $C$ by (4.7) and $P \pi$ by $(4.16)_{2}$, the force balance (4.1) is satisfied. We may therefore restrict attention to (4.11) and (4.12) with the assurance that the force balance (4.1) can always be satisfied.

3. By (4.7), C is characterized by the vector

$$
\boldsymbol{\xi}=\sigma \mathbf{m}-\mathbf{c}
$$

of Cahn and Hoffman $[1972,1974]$ in the sense that

$$
\mathbf{C}=(\boldsymbol{\xi} \cdot \mathbf{m}) \mathbf{P}-\mathbf{m} \otimes \boldsymbol{\xi} .
$$




\section{Thermodynamical laws.} form: ${ }^{11}$

a. Balance of energy. Growth of entropy. We consider the first two laws in the balance of energy

$$
\left\{\int_{R} \varepsilon+\int_{\nu} e\right\}^{\cdot}=-\int_{\partial R} \mathbf{q} \cdot \mathbf{n}+\int_{\partial \nu}\left(h W_{\nu}+\mathbf{C} \boldsymbol{\nu} \cdot \mathbf{w}_{\nu}\right)
$$

growth of entropy

$$
\left\{\int_{R} \eta+\int_{\nu} s\right\}^{\cdot} \geq-\int_{\partial R}(\mathbf{q} / \vartheta) \cdot \mathbf{n}+\int_{\partial \nu}(h / \vartheta) W_{\nu}
$$

for all control volumes $R$, where $\mathbf{n}$ is the outward unit normal to $\partial R$. The terms $h W$, and $(h / \vartheta) W$, represent flows of heat and entropy into $r$ across $\partial r$ induced by the tangential motion of $\partial \mu$. Our analysis will show that these terms cannot vanish if the interface is endowed with entropy.

Restricting attention to control volumes that do not intersect the interface leads to the standard bulk relations

$$
\dot{\varepsilon}=-\operatorname{div} \mathbf{q}, \quad \dot{\eta} \geq-\operatorname{div}(\mathbf{q} / \vartheta),
$$

which combine to form the bulk dissipation inequality

$$
\dot{\psi}+\eta \dot{\vartheta}-\vartheta^{-1} \mathbf{q} \cdot \nabla \vartheta \leq 0 .
$$

On the other hand, if we let $R$ be a facet control volume at some arbitrarily chosen time $t_{0}$, with $F(t)$ the underlying facet, then, by (2.8), (2.9), (4.9), and (4.13), if we shrink $R$ to the interface in (5.1) and (5.2), we find that

$$
\begin{gathered}
\int_{\nu}\{\stackrel{\circ}{e}+(\pi-[\varepsilon]) V+[\mathbf{q}] \cdot \mathbf{m}\}=\int_{\partial \nu}(h+\sigma-e) W_{\nu}, \\
\left.\int_{\nu}\{\stackrel{o}{s}-[\eta]) V+\vartheta^{-1}[\mathbf{q}] \cdot \mathbf{m}\right\} \geq \int_{\partial \nu}\left(\vartheta^{-1} h-s\right) W_{\nu} .
\end{gathered}
$$

Given any sufficiently regular subset $r_{0}$ of $F\left(t_{0}\right)$, and any smooth scalar field $\varphi$ on $\partial \mu_{0}$, we can always find a control volume $R$ such that $\mu\left(t_{0}\right)=\mu_{0}$ and $W_{\mu}\left(\mathbf{x}, t_{0}\right)=$ $\varphi(\mathbf{x})$ on $\partial r_{0}$. Therefore,

$$
\sigma=f, \quad h=\vartheta s,
$$

and we have the classical identification of surface tension with interfacial free energy. By (5.1), $d Q=h W, d s$ represents the rate of apparent heating of an element of length $d s$ on $\partial \mu$; thus writing $d S=s W, d s$ for the rate at which entropy is transferred across $d s$ as it moves tangentially, then the second of (5.6) is the classical relation $d Q=\vartheta d S$.

Next, since $r$ is arbitrary, (5.5) and (5.6) yield localizations

$$
\stackrel{\circ}{e}+(\pi-[\varepsilon]) V=-[\mathbf{q}] \cdot \mathbf{m}, \quad \stackrel{\circ}{s}-[\eta] V \geq-\vartheta^{-1}[\mathbf{q}] \cdot \mathbf{m}
$$

${ }^{11}$ Cf. Gurtin and Struthers [1990], Gurtin [1991]. 
of the first two laws to the interface; these combine to form the interfacial dissipation inequality

$$
\stackrel{\circ}{f}+s \stackrel{\vartheta}{\vartheta}+(\pi-[\psi]) V \leq 0 .
$$

The results (5.7) and (5.8) are valid on each facet, but not generally on edges (across which the interfacial fields may suffer jump discontinuities).

We turn next to a discussion of the edges. Choose adjacent facets $F(t)$ and $G(t)$, let $R$ be an edge control volume for the edge $F(t) \cap G(t)$, and let $z(t)$ denote the intersection of $R$ with $F(t) \cap G(t)$. The quantity

$$
\mathscr{E}(R)=\left\{\int_{R} \varepsilon\right\}^{\cdot}+\int_{\partial R} \mathbf{q} \cdot \mathbf{n}
$$

represents the bulk energy production in $R$. If the bulk fields $\varepsilon$ and $\mathbf{q}$ were well behaved at the edges, then $\mathscr{E}(R)$ would vanish as $R$ shrinks to $z(t)$. We now show that, because of the presence of interfacial structure, this will generally not be so.

By $(5.1)$,

$$
\mathscr{E}(R)=-\left\{\int_{\mu} e\right\}^{\cdot}+\int_{\partial r}\left(h W_{\nu}+\mathbf{C} \nu \cdot \mathbf{w}_{\nu}\right) .
$$

Since $r(t)$ is contained in the union of $F(t)$ and $G(t)$, we may use (2.3), (2.9), and (4.5) to conclude that

$$
\left\{\int_{\nu^{\prime}} e\right\}^{\cdot}=\left\{\int_{\nu_{F}} e+\int_{\nu_{G}} e\right\}^{\cdot}=\left\{\int_{\nu_{F}} \stackrel{\circ}{e}+\int_{\nu_{G}} \stackrel{\circ}{e}\right\}+\left\{\int_{\partial_{\nu_{F}}} e W_{\nu_{F}}+\int_{\partial \nu_{G}} e W_{\mu_{G}}\right\},
$$

and, furthermore, the last term $\{\cdots\}$ may be written as

$$
\int_{\partial,} e W_{r}+\int_{z}\left(e_{F} W_{F}+e_{G} W_{G}\right)
$$

Next, by (4.6) and (4.9),

$$
\begin{aligned}
\int_{\partial \nu^{\prime}} \mathbf{C} \boldsymbol{\nu} \cdot \mathbf{w}_{\mu}=\int_{\partial \nu} & \sigma W_{\mu}+\int_{z}\left(\sigma_{F} W_{F}+\sigma_{G} W_{G}\right) \\
& +\int_{\partial \nu_{F}} V_{F} \mathbf{c} \cdot \nu_{\nu_{F}}+\int_{\partial r_{G}} V_{G} \mathbf{c} \cdot \nu_{r_{G}},
\end{aligned}
$$

and, by $(4.16)_{1}$, the final two terms reduce to

$$
-\int_{,} \pi V .
$$

Combining (5.10)-(5.14), and using (5.6), we find that

$$
\mathscr{E}(R)=-\int_{x} \vartheta\left(s_{F} W_{F}+s_{G} W_{G}\right)-\left\{\int_{\nu_{F}} \stackrel{\circ}{e}+\int_{\nu_{G}} \stackrel{\circ}{e}\right\}-\int_{\nu} \pi V .
$$

Therefore, letting $R$ shrink to $\approx$ with $\operatorname{area}(\mu) \rightarrow 0$, we find that

$$
\mathscr{E}(R) \rightarrow-\int_{\mathfrak{x}} \vartheta\left(s_{F} W_{F}+s_{G} W_{G}\right)
$$


thus at each edge there is a net production of bulk energy induced by the tangential speeds of the facet boundaries in the presence of interfacial entropy. Note that, by (5.6), the integrand in (5.16) is $\left(h_{F} W_{F}+h_{G} W_{G}\right)$, so that the production of bulk energy at an edge is balanced by the apparent heating of the two facets at the edge.

$b$. Second law in terms of a Gibbs function. ${ }^{12}$ We will be most interested in situations involving small departures from a given constant temperature $\vartheta_{T}$. The physical interpretation of $\vartheta_{T}$ is irrelevant to the discussion of this section; in later sections it will denote the transition temperature. Dynamics with small temperature changes is cumbersome using a formulation based on entropy; a more useful version of the second law involves the Gibbs function

$$
\bar{\varphi}=\varepsilon-\vartheta_{T} \eta
$$

in conjunction with the field

$$
\bar{u}=\left(\vartheta-\vartheta_{T}\right) / \vartheta
$$

and is derived by multiplying (5.2) by $\vartheta_{T}$ and subtracting the resulting inequality from (5.1); the result is

$$
\left\{\int_{R} \bar{\varphi}+\int_{\nu}\left(e-\vartheta_{T} s\right)\right\}^{\cdot} \leq-\int_{\partial R} \bar{u} \mathbf{q} \cdot \mathbf{n}+\int_{\partial \nu}\left(\bar{u} h W_{\nu}+\mathbf{C} \boldsymbol{\nu} \cdot \mathbf{w}_{\nu}\right) .
$$

\section{Constitutive equations.}

a. Bulk constitutive equations. We consider bulk constitutive equations for the entropy and free energy of each phase $\mu=\alpha, \beta$ in the standard form

$$
\eta=\hat{\eta}_{\mu}(\vartheta), \quad \psi=\hat{\psi}_{\mu}(\vartheta),
$$

with constitutive response functions $\hat{\eta}_{\mu}$ and $\hat{\psi}_{\mu}$ related through

$$
\hat{\eta}_{\mu}(\vartheta)=-d \hat{\psi}_{\mu}(\vartheta) / d \vartheta
$$

To these we adjoin a Fourier law for the heat flux:

$$
\mathbf{q}=-\mathbf{K}_{\mu}(\vartheta) \nabla \vartheta
$$

with $\mathbf{K}_{\mu}(\vartheta)$, the conductivity tensor, positive definite. The relations (6.2) and (6.3) ensure satisfaction of the bulk dissipation inequality (5.4).

In view of (3.2), (6.1) yield an analogous constitutive equation

$$
\varepsilon=\hat{\varepsilon}_{\mu}(\vartheta)
$$

for the internal energy, whose derivative

$$
c_{\mu}(\vartheta)=d \hat{\varepsilon}_{\mu}(\vartheta) / d \vartheta
$$

is the bulk specific heat.

In classical theories of solidification there is a temperature $\vartheta_{T}$, called the transition temperature, at which the phase transition takes place. At $\vartheta_{T}$ the free energies of the two phases coincide and $\hat{\psi}_{\alpha}(\vartheta)-\hat{\psi}_{\beta}(\vartheta)$ changes sign, indicating a change in the relative stability of the two phases. Here we suppose that a transition temperature exists, but we do not require that the phase change take place at $\vartheta_{T}$.

${ }^{12}$ Cf. Gurtin [1933b], $\S 3$. 
Precisely, we assume that there is a unique temperature $\vartheta_{T}$, called the transition temperature, at which

$$
\hat{\psi}_{c}\left(\vartheta_{T}\right)=\hat{\psi}_{\beta}\left(\vartheta_{T}\right)=0
$$

where without loss of generality we have set to zero this common value of the free energy. The difference

$$
\ell=\hat{\varepsilon}_{\beta}\left(\vartheta_{T}\right)-\hat{\varepsilon}_{\alpha}\left(\vartheta_{T}\right)
$$

in internal energy between phases at the transition temperature is the latent heat, which we assume to be nonzero. To discuss behavior near the transition temperature we introduce the temperature difference

$$
u=\left(\vartheta-\vartheta_{T}\right) / \vartheta_{T}
$$

then, for $|u|$ small,

$$
\begin{aligned}
\hat{\psi}_{\beta}(\vartheta)-\hat{\psi}_{\alpha}(\vartheta) & =-\ell u+O\left(u^{2}\right), \\
\hat{\varepsilon}_{\beta}(\vartheta)-\hat{\varepsilon}_{\alpha}(\vartheta) & =\ell+O(u),
\end{aligned}
$$

and, for $\bar{\varphi}$ and $\bar{u}$ defined by (5.17) and (5.18),

$$
\begin{aligned}
& \bar{u}=u+O\left(u^{2}\right), \\
& \bar{\varphi}=\vartheta_{T} c_{\mu}\left(\vartheta_{T}\right) u^{2}+O\left(u^{3}\right) \quad \text { in phase } \mu, \\
& \psi=\bar{\varphi}-u \varepsilon+O\left(u^{3}\right) .
\end{aligned}
$$

$b$. Interfacial constitutive equations. We now restrict attention to evolutions of the body for which

the orientation $\mathbf{m}$ of the interface is confined to a finite set $\mathscr{M}$.

$\mathscr{M}$ is related to the lattice structure of the crystal and should be envisaged as representing stable orientations of the interface. As constitutive equations we allow the free energy $f$, the entropy $s$, and the normal interactive force $\pi$ to depend, not only on the temperature $\vartheta$, but also on the orientation and kinetics of the interface through dependences on $\mathbf{m} \in \mathscr{M}$ and $V$ :

$$
f=\hat{f}(\vartheta, \mathbf{m}, V), \quad s=\hat{s}(\vartheta, \mathbf{m}, V), \quad \pi=\hat{\pi}(\vartheta, \mathbf{m}, V) .
$$

We require that these constitutive relations be consistent with the interfacial dissipation inequality (5.8). Then for $F(t)$ a facet with orientation $\mathbf{m} \in \mathscr{M}$,

$$
\left\{\hat{f}_{\vartheta}(\vartheta, \mathbf{m}, V)+\hat{s}(\vartheta, \mathbf{m}, V)\right\} \stackrel{\circ}{\vartheta}+\hat{f}_{V}(\vartheta, \mathbf{m}, V) \stackrel{\circ}{V}+\{\hat{\pi}(\vartheta, \mathbf{m}, V)-[\psi]\} V \leq 0,
$$

with $[\psi]=\hat{\psi}_{\beta}(\vartheta)-\hat{\psi}_{c x}(\vartheta)$, and (6.13) holds for all such facets and for all timedependent temperature fields if and only if

(i) $\hat{f}(\vartheta, \mathbf{m}, V)$ and $\hat{s}(\vartheta, \mathbf{m}, V)$ are independent of $V$, and

$$
\hat{s}(\vartheta, \mathbf{m})=-\hat{f}_{\vartheta}(\vartheta, \mathbf{m})
$$

(ii) there is a kinetic modulus $\hat{b}(\vartheta, \mathbf{m}, V) \geq 0$ such that

$$
\hat{\pi}(\vartheta, \mathbf{m}, V)=\hat{\psi}_{\beta}(\vartheta)-\hat{\psi}_{\alpha}(\vartheta)-\hat{b}(\vartheta, \mathbf{m}, V) V .
$$


We assume, henceforth, that (6.14) and (6.15) are satisfied with

$$
\hat{f}(\vartheta, \mathbf{m})>0, \quad \hat{b}(\vartheta, \mathbf{m}, V)>0 .
$$

\section{The general free-boundary problem.}

a. Bulk equations. Interface conditions. The equations derived thus far combine to form a free-boundary problem for the temperature. The bulk equations consist of the energy balance

$$
\dot{\varepsilon}=-\operatorname{div} \mathbf{q}
$$

supplemented by the constitutive equations

$$
\psi=\hat{\psi}_{\mu}(\vartheta), \quad \eta=-d \hat{\psi}_{\mu}(\vartheta) / d \vartheta, \quad \varepsilon=\psi+\vartheta \eta, \quad \mathbf{q}=-\mathbf{K}_{\mu}(\vartheta) \nabla \vartheta
$$

in each phase $\mu=\alpha, \beta$, which combine to give the partial differential equation

$$
c_{\mu}(\vartheta) \dot{\vartheta}=\operatorname{div}\left\{\mathbf{K}_{\mu}(\vartheta) \nabla \vartheta\right\}
$$

in each phase.

The interface conditions consist of (6.11), the energy balance

$$
[\varepsilon] V=[\mathbf{q}] \cdot \mathbf{m}+\stackrel{\circ}{e}+\pi V
$$

on $\mathscr{S}$, and the force balance relations (4.11) and (4.12) with $\sigma$ replaced by $f$. These interface conditions are supplemented by the constitutive equations

$$
f=\hat{f}(\vartheta, \mathbf{m}), \quad s=-\hat{f}_{\vartheta}(\vartheta, \mathbf{m}), \quad \pi=\hat{\pi}(\vartheta, \mathbf{m}, V), \quad \pi=[\psi]-\hat{b}(\vartheta, \mathbf{m}, V) V .
$$

Defining

$$
\lambda_{F(i}(\vartheta)=\left\{\left(\mathbf{m}_{F} \cdot \mathbf{m}_{C_{i}}\right) \hat{f}\left(\vartheta, \mathbf{m}_{F}\right)-\hat{f}\left(\vartheta, \mathbf{m}_{C_{i}}\right)\right\} /\left\{1-\left(\mathbf{m}_{F} \cdot \mathbf{m}_{C_{i}}\right)^{2}\right\}^{1 / 2},
$$

we can rewrite $(4.11)$ and $(4.12)$ as

$$
\begin{aligned}
& \left.\int_{F^{*}}[\psi](\mathbf{x}, t) d A(\mathbf{x})=-\sum_{\sigma_{i}}\left\{\int_{F \cap C_{i}} \lambda_{F(i}(\vartheta(\mathbf{x}, t)) d\right\lrcorner(\mathbf{x})\right\} \\
& +V_{F^{\prime}}(t) \int_{r^{\prime}} \hat{b}\left(\vartheta(\mathbf{x}, t), \mathbf{m}_{F^{*}}, V_{F^{\prime}}(t)\right) d A(\mathbf{x}),
\end{aligned}
$$

where the sum is over all facets $G$ adjacent to $F$, and where $d A$ and $d s$ are the elements of area and length. Since $[\psi]$ is a function of the temperature, (7.7) may be viewed as a relation between the normal speed $V_{F}(t)$ of the facet $F(t)$ and the temperature field over the entire facet.

There is also the restriction (5.16) on the strength of the singularity in the bulk fields at the edges.

b. Initial conditions. Boundary conditions. Appropriate initial conditions involve the prescription of

$$
\Omega_{\text {, }}(0) \text { and } \vartheta(\mathbf{x}, 0) \text { for all } \mathbf{x} \in \mathbb{R}^{3} \text {. }
$$

If, as assumed, the body (the region of space occupied by the two phases) is all of $\mathbb{R}^{3}$, conditions at infinity are needed; these are standard if the interface is finite. If the body $\Omega=\Omega_{11}(t) \cup \Omega_{\beta}(t)$ is a bounded region, then boundary conditions are required. 
When the interface $\mathscr{S}(t)$ touches the boundary, conditions expressing balance of capillary forces are needed at the juncture of the interface and the boundary. Here we will restrict attention to situations in which the interface does not touch the boundary. Appropriate boundary conditions are then a prescription of

$\vartheta(\mathbf{x}, t)$ on a portion of $\partial \Omega$ and $\mathbf{q}(\mathbf{x}, t) \cdot \mathbf{n}(\mathbf{x})$ on the remainder, with $\mathbf{n}(\mathbf{x})$ the outward unit normal to $\partial \Omega$,

and the free-boundary problem consists of the bulk equations (7.3), the free-boundary conditions (6.11), (7.4), (7.6), and (7.7), the singularity restriction (5.16), the initial conditions (7.8), and the boundary conditions (7.9). One might also add an admissibility condition of the type discussed in Sec. 8c.

\section{Behavior near the transition temperature.}

a. The quasi-linear system. We now linearize the bulk and interface equations in the variables $u$ and $V$, neglecting the term $\stackrel{\circ}{e}$ in the energy balance (7.4). We shall simply omit nonlinear terms, a precise asymptotic development being beyond the scope of the paper. However, because of the free boundary, the resulting system of equations will remain nonlinear.

It is convenient to write

$$
\hat{f}(\mathbf{m})=\hat{f}\left(\vartheta_{T}, \mathbf{m}\right), \quad \hat{b}(\mathbf{m})=\hat{b}\left(\vartheta_{T}, \mathbf{m}, 0\right),
$$

and to let $L_{F G}(t)$ and $A_{F}(t)$, respectively, denote the length of the edge $F(t) \cap G(t)$ and the area of the facet $F(t)$. Then, guided by (6.9), we replace (7.4) and (7.7) by the interface conditions

$$
\begin{aligned}
& \ell V=[\mathbf{q}] \cdot \mathbf{m}, \\
& \ell \int_{F} u(\mathbf{x}, t) d A(\mathbf{x})=\sum_{G} \lambda_{F G} L_{F G}(t)-A_{F}(t) \hat{b}\left(\mathbf{m}_{F}\right) V_{F}(t),
\end{aligned}
$$

where the first of $(8.2)$ is to hold on $\mathscr{S}$, the second is to hold for each facet $F$, the sum is over all facets $G$ adjacent to $F$, and $\lambda_{F G}$ are now the constants

$$
\lambda_{F G}=\left\{\left(\mathbf{m}_{F} \cdot \mathbf{m}_{G}\right) \hat{f}\left(\mathbf{m}_{F}\right)-\hat{f}\left(\mathbf{m}_{G}\right)\right\} /\left\{1-\left(\mathbf{m}_{F} \cdot \mathbf{m}_{G}\right)^{2}\right\}^{1 / 2} .
$$

Note that, in contrast to the classical Stefan condition $u=0$, the second of (8.2) represents a condition on the integral of $u$ over each facet $F$.

Similarly, writing

$$
c_{\mu} \text { for } c_{\mu}\left(\vartheta_{T}\right) \vartheta_{T}, \quad \mathbf{K}_{\mu} \text { for } \mathbf{K}_{\mu}\left(\vartheta_{T}\right) \vartheta_{T},
$$

we replace (7.3) by the bulk equations

$$
c_{\mu} \dot{u}=-\operatorname{div} \mathbf{q}, \quad \mathbf{q}=-\mathbf{K}_{\mu} \nabla u .
$$

We will use the term quasi-linear system to denote the bulk equations (8.5) in each phase $\mu$ supplemented by the interface conditions (6.11) and (8.2). The freeboundary problem associated with this system is obtained by adjoining the initial and boundary conditions (7.8) and (7.9) (with $\vartheta$ replaced by $u$ ). (We view the singularity condition (5.16) as inappropriate for this system. In the next section we 
show that for $c_{\alpha}=c_{\beta}$ the energy balance (9.11) is satisfied; this balance, when taken as basic, yields, in place of $(5.16)$, the conclusion $\mathscr{E}(R) \rightarrow 0$.)

b. Frank diagram. Convexified energy. The Frank diagram ${ }^{13} \mathscr{F}$ (at the transition temperature) is the boundary of the convex hull of the finite set

$$
\mathscr{G}=\left\{\hat{f}(\mathbf{m})^{-1} \mathbf{m}: \mathbf{m} \in \mathscr{M}\right\} .
$$

$\mathscr{F}$ is a polyhedral surface whose vertices belong to $\mathscr{G}$; we will, in fact, assume that

$$
\mathscr{G} \text { is the exact set of vertices of } \mathscr{F} \text {, }
$$

an assumption ensuring that the Wulff shape corresponding to $\hat{f}(\mathbf{m}), \mathbf{m} \in \mathscr{M}$, have $\mathscr{M}$ as its set of orientations. The construction of $\mathscr{F}$ ensures that $\mathscr{F}$ correspond to an "energy" $\hat{f}_{0}(\mathbf{m})$ defined for all $\mathbf{m} \in S^{2}$ :

$$
\mathscr{F}=\left\{\hat{f}_{0}(\mathbf{m})^{-1} \mathbf{m}: \mathbf{m} \in S^{2}\right\},
$$

where $S^{2}$ is the set of all unit vectors. The function $\hat{f}_{0}$ extends $\hat{f}$ from $\mathscr{M}$ to $S^{2}$. It is convenient to further extend $\hat{f}$ to $\mathbb{R}^{3}$ by homogeneity: $f_{0}(\mathbf{0})=0$ and

$$
f_{0}(\mathbf{p})=|\mathbf{p}| \hat{f}_{0}(\mathbf{p} /|\mathbf{p}|)
$$

for all $\mathbf{p} \in \mathbb{R}^{3}, \mathbf{p} \neq \mathbf{0}$. Then $\mathscr{F}$ is the one-level set of $f_{0}$, so that $f_{0}$ is a convex function. We will refer to $f_{0}$ as the convexified energy.

c. Admissible interfaces. ${ }^{14}$ Both the Frank diagram and the crystal are polyhedral; to avoid confusion we will use the following differences in terminology:

$\begin{array}{ll}\text { crystal } & \text { Frank diagram } \\ \text { facet } & \text { face } \\ \text { corner } & \text { vertex } .\end{array}$

We will refer to $\mathscr{P} \subset \mathscr{M}$ as compatible if there is a face $f$ of $\mathscr{F}$ such that $\hat{f}(\mathbf{m})^{-1} \mathbf{m}$ is a vertex of $f$ for each $\mathbf{m} \in \mathscr{P}$. We will refer to $\mathbf{m}, \mathbf{r} \in \mathscr{M}$ as adjacent if the line segment from $\hat{f}(\mathbf{m})^{-1} \mathbf{m}$ to $\hat{f}(\mathbf{r})^{-1} \mathbf{r}$ is an edge of $\mathscr{F}$ (in which case $\{\mathbf{m}, \mathbf{r}\}$ is compatible).

For the interfaces $\mathscr{S}(t)$ under discussion the orientation of each facet belongs to $\mathscr{M}$ (cf. (6.11)). We now consider two additional conditions:

(W1) orientations of adjacent facets are adjacent orientations;

(W2) each set-of orientations of facets that intersect at a corner-is compatible.

We will refer to $\mathscr{S}$ as admissible if $\mathscr{S}$ is consistent with (W1) and (W2); we assume, for the remainder of this section, that $\mathscr{S}$ is admissible.

RemarKs. 1. (W1) and (W2) are satisfied by the Wulff shape; in fact, they are equivalent to the conditions: (i) orientations of adjacent facets are orientations of adjacent facets on the Wulff shape; (ii) the complete set of orientations of facets

\footnotetext{
${ }^{13}$ Frank [1963]. Cf. Angenent and Gurtin [1989] for a detailed discussion.

${ }^{14}$ This section is taken from Giga, Gurtin, and Matias [1993].
} 
meeting in any given corner is a complete set also for some corner of the Wulff shape.

2. Admissibility is at least plausible. Almgren and Taylor [1992] consider the problem of evolution from an admissible interface in $\mathbb{R}^{2}$ within the framework of (9.38) with $D=0$, but with a general crystalline energy whose domain is $S^{2}$ rather than $\mathscr{M}$ and whose Wulff shape has $\mathscr{M}$ as its set of orientations. They give a variational formulation of this problem that allows for all orientations in $S^{2}$ and show that its solution $\mathscr{S}$ has orientations in $\mathscr{M}$, evolves according to (9.38) with $D=0$, and, what is most important, is admissible.

The conditions (W1) and (W2) establish a correspondence between corners on the interface and faces on the Frank diagram. Precisely, if $c$ is a corner of $\mathscr{S}$, and if $\mathscr{P}$ is the set of orientations of facets that intersect at $c$, then there is a unique face

$$
f=\hat{f}(c)
$$

of the Frank diagram such that

$$
\mathscr{L}=\left\{\hat{f}(\mathbf{m})^{-1} \mathbf{m}: \mathbf{m} \in \mathscr{P}\right\}
$$

is the set of vertices of $\mathcal{f}$. Indeed, by (W2), $\mathscr{L}$ is contained in the set $\mathscr{V}$ of vertices of a face $f$ of $\mathscr{F}$, and, in view of (W1), $\mathscr{V}=\mathscr{L}$.

The energy $f_{0}(\mathbf{p})$ is differentiable at all $\mathbf{p}$ with $\mathbf{p} /|\mathbf{p}| \notin \mathscr{M}$; in fact, given any face $f$ on the Frank diagram, $\nabla f_{0}$ is constant on the cone

$$
\{\alpha \mathbf{p}: \mathbf{p} \in \mathcal{f}, \mathbf{p} /|\mathbf{p}| \notin \mathscr{M}, \alpha>0\} .
$$

We denote this constant by $\nabla f_{0}(f)$. The next result shows that the $\lambda$ 's in (8.3) are completely determined by the gradient $\nabla f_{0}$. Precisely, we will show that if $F$ and $G$ are adjacent facets and if $c$ is a corner terminating the edge $F \cap G$, then ${ }^{15}$

$$
\lambda_{F G}=\nu_{F} \cdot \nabla f_{0}(\hat{f}(c))
$$

with $\nu_{F}$ the outward unit normal to $\partial F$ on $F \cap G$.

To verify (8.13), let $f=\hat{f}(c)$, and let $\mathscr{C}$ denote the intersection of $S^{2}$ with the set (8.12). Then $\nabla f_{0}(f)=\nabla f_{0}(\mathbf{m})$ for any $\mathbf{m} \in \mathscr{C}$, and, by homogeneity, $\mathbf{m} \cdot \nabla f_{0}(\mathbf{m})=\hat{f}_{0}(\mathbf{m})$ for $\mathbf{m} \in \mathscr{C}$. Thus, given any $\mathbf{m} \in \mathscr{C}$, we may write $\nabla f_{0}(f)$ in the form

$$
\nabla f_{0}(f)=\hat{f}_{0}(\mathbf{m}) \mathbf{m}-\hat{\mathbf{c}}(\mathbf{m}), \quad \hat{\mathbf{c}}(\mathbf{m}) \cdot \mathbf{m}=0,
$$

and, by continuity, (8.14) holds also for $\mathbf{m}=\mathbf{m}_{F}, \mathbf{m}_{G}$.

Next, let $\nu_{F}$ and $\nu_{G}$ denote the outward unit normals to $\partial F$ and $\partial G$ on $F \cap G$. Then $\boldsymbol{\nu}_{F}$ and $\boldsymbol{\nu}_{G}$ lie in the plane spanned by $\mathbf{m}_{F}$ and $\mathbf{m}_{G}$ with $\boldsymbol{\nu}_{F} \times \mathbf{m}_{F}=-\boldsymbol{\nu}_{G} \times \mathbf{m}_{G}$, and therefore

$$
\mathbf{m}_{F} \otimes \boldsymbol{\nu}_{F}-\boldsymbol{\nu}_{F} \otimes \mathbf{m}_{F}=-\left[\mathbf{m}_{G} \otimes \boldsymbol{\nu}_{G}-\boldsymbol{\nu}_{G} \otimes \mathbf{m}_{G}\right]
$$

(the left side acting on a vector $\mathbf{v}$ is $\pm\left(\nu_{F} \times \mathbf{m}_{F}\right) \times \mathbf{v}$; the right side is also, with the same sign). Thus, applying the left side of (8.15) to (8.14) at $\mathbf{m}=\mathbf{m}_{F}$ and the right

\footnotetext{
${ }^{15}$ Ben Amar and Pomeau [1988] and Gurtin and Matias [1990] establish an analogous condition for the interface a polygonal curve in the plane.
} 
side at $\mathbf{m}=\mathbf{m}_{G}$, we conclude that (4.10) holds with $\sigma_{E}=\hat{f}\left(\mathbf{m}_{E}\right)$ and $\mathbf{c}_{E}=\hat{\mathbf{c}}\left(\mathbf{m}_{E}\right)$, $E=F, G$. Thus (4.11) and hence (8.3) is satisfied, and, since $\nabla f_{0}(f) \cdot \nu_{F}=\mathbf{c}_{F} \cdot \boldsymbol{\nu}_{F}$, this completes the proof.

9. Thermodynamically consistent theory for behavior near the transition temperature.

a. Modified quasi-linear system. The quasi-linear system is an approximation of the general theory and cannot be expected to obey the general laws of energy balance and entropy growth. For example, when $c_{\alpha} \neq c_{\beta}$ the interface condition given by the first of (8.2) is not the appropriate jump condition for the energy equation (7.1), even when the interface is devoid of internal energy. We now modify the quasilinear system by adding "higher-order" terms that give the theory an approximate thermodynamic structure.

We introduce the bulk internal energy

$$
\varepsilon(\mathbf{x}, t)= \begin{cases}c_{\alpha} u(\mathbf{x}, t), & \mathbf{x} \in \Omega_{\alpha}(t) \\ \ell+c_{\beta} u(\mathbf{x}, t), & \mathbf{x} \in \Omega_{\beta}(t),\end{cases}
$$

the bulk free energy

$$
\psi(\mathbf{x}, t)= \begin{cases}-\frac{1}{2} c_{\alpha} u(\mathbf{x}, t)^{2}, & \mathbf{x} \in \Omega_{\alpha}(t) \\ -\ell u-\frac{1}{2} c_{\beta} u(\mathbf{x}, t)^{2}, & \mathbf{x} \in \Omega_{\beta}(t),\end{cases}
$$

and the Gibbs function (cf. $\left.(6.10)_{3}\right)$

$$
\varphi(\mathbf{x}, t)=\psi+u \varepsilon= \begin{cases}\frac{1}{2} c_{\alpha} u(\mathbf{x}, t)^{2}, & \mathbf{x} \in \Omega_{\alpha}(t) \\ \frac{1}{2} c_{\beta} u(\mathbf{x}, t)^{2}, & \mathbf{x} \in \Omega_{\beta}(t) ;\end{cases}
$$

and we replace $(8.2)$ by

$$
\begin{aligned}
{[\varepsilon] V } & =[\mathbf{q}] \cdot \mathbf{m}, \\
-\int_{F}[\psi] & =\sum_{G} \lambda_{F G} L_{F G}-A_{F} \hat{b}\left(\mathbf{m}_{F}\right) V_{F} .
\end{aligned}
$$

We refer to (6.11), (8.5), (9.2), and (9.4) as the modified quasi-linear system. ${ }^{16}$ Note that

$$
[\varepsilon]=\ell+[c] u, \quad[\psi]=-\ell u-\frac{1}{2}[c] u^{2},
$$

so that (9.4) differ from (8.2) only in the presence of the higher-order terms $[c] u$ in the first of (9.4) and $-\frac{1}{2}[c] u^{2}$ in the second. Thus, in particular,

the interface conditions (9.4) of the modified quasi-linear system reduce to the conditions (8.2) when the specific heats of the two phases coincide.

It is important to note that $\varepsilon$ and $\psi$ do not represent the actual bulk internal and free energies but rather their approximations near the transition temperature.

\footnotetext{
${ }^{16}$ We view the singularity condition (5.16) as inappropriate for this system. One might also wish to require that $\mathscr{S}$ be admissible; the ensuing results are independent of such an assumption.
} 
Consider now a solution of the modified quasi-linear system. We introduce an interfacial free energy $f(\mathbf{x}, t)$ and a kinetic modulus $b(\mathbf{x}, t)$, defined on each facet $F$ by

$$
f(\mathbf{x}, t)=\hat{f}\left(\mathbf{m}_{F}\right), \quad b(\mathbf{x}, t)=\hat{b}\left(\mathbf{m}_{F}\right), \quad \mathbf{x} \in F(t) ;
$$

$f$ and $b$ approximate the actual interfacial free energy and kinetic modulus near the transition temperature.

We also introduce a surface shear $\mathbf{c}(\mathbf{x}, t)$ defined on each facet $F$ as the solution of the boundary-value problem:

$$
\begin{array}{ll}
\operatorname{div}_{F} \mathbf{c}+\pi=0 & \text { on } F, \\
\mathbf{c}_{F} \cdot \nu_{F}=\lambda_{F G} & \text { on each of the edges } F \cap G \text { of } F,
\end{array}
$$

with $\lambda_{F G}$ given by (8.3) and

$$
\pi=[\psi]-b V .
$$

(The second of (9.4) implies (7.7), and this ensures that (9.8) has a solution.) Finally, we define a capillary force

$$
\mathbf{C}=f \mathbf{P}+\mathbf{m} \otimes \mathbf{c} .
$$

Then reasoning as in Remark 2 following (4.16), we see that $\mathrm{C}$ satisfies the force balance (4.1) (with $\pi$ defined by the second of (4.16) and (9.9)).

We now show that solutions of the modified quasi-linear system satisfy the energy balance

$$
\left\{\int_{R} \varepsilon\right\}^{\cdot}=-\int_{\partial R} \mathbf{q} \cdot \mathbf{n}
$$

and the dissipation inequality ${ }^{17}$

$$
\left\{\int_{R} \varphi+\int_{r} f\right\}^{\cdot} \leq-\int_{\partial R} u \mathbf{q} \cdot \mathbf{n}+\int_{\partial,} \mathbf{C} \boldsymbol{\nu} \cdot \mathbf{w}_{\nu}
$$

for each control volume $R$, with the difference between the right and left sides of (9.12) given by

$$
\mathscr{D}(u, R):=\int_{\mu} b V^{2}+\sum_{\mu} \int_{R_{\mu}} \nabla u \cdot \mathbf{K}_{\mu} \nabla u,
$$

the sum being over $\mu=\alpha, \beta$.

The verification of (9.11) and (9.12) is based on two identities. The first, a direct consequence of the divergence theorem, asserts that

$$
\sum_{\mu} \int_{R_{\mu}} \operatorname{div} \mathbf{k} d a=\int_{\partial R} \mathbf{k} \cdot \mathbf{n}-\int_{\nu}[\mathbf{k}] \cdot \mathbf{m}
$$

for any bulk vector field $\mathbf{k}$. The second identity is

$$
\left\{\int_{\nu} f\right\}^{\cdot}=\int_{\nu} \pi V+\int_{\partial \nu} \mathbf{C} \boldsymbol{\nu} \cdot \mathbf{w}_{\nu} .
$$

${ }^{17}$ Cf. Gurtin [1993b], Eq. (3.3). 
To establish (9.15), we note that, since $f$ is constant on each facet, we may use (2.9) and $(9.10)$ to conclude that

$$
\begin{aligned}
\left\{\int_{\nu} f\right\}^{\cdot} & =\sum_{F} \int_{\partial_{r_{F}}} f_{F} W_{F}=\sum_{F} \int_{\mathscr{R}_{F}} f_{F} W_{F}+\int_{\partial \nu} f W \\
& =\sum_{F} \int_{\mathscr{R}_{F}} f_{F} W_{F}+\int_{\partial \nu}\left(\mathbf{C} \boldsymbol{\nu}_{\nu} \cdot \mathbf{w}_{\nu}-V \mathbf{c} \cdot \boldsymbol{\nu}\right),
\end{aligned}
$$

where $\mathscr{R}_{F}(t)=\partial \mu_{F}(t) \backslash \partial \mu(t)$ and the sum is over all facets $F$ with $r_{F}(t)=r(t) \cap$ $F(t)$ not empty. Next, since $V=V_{F}$ on each facet $F$,

$$
\int_{\partial r} V \mathbf{c} \cdot \boldsymbol{\nu}=-\sum_{F} \int_{\mathscr{R}_{F}} V_{F} \mathbf{c} \cdot \nu_{r_{F}}+\int_{r^{\prime}} V \operatorname{div}_{F} \mathbf{c} .
$$

Further, since $C$ satisfies (4.1), (4.3) holds on each edge $F \cap G$, and, by (4.3) and (9.10),

$$
\sum_{F} \int_{\mathscr{R}_{F}}\left(f_{F} W_{F}+V_{F} \mathbf{c} \cdot \boldsymbol{\nu}_{\nu_{F}}\right)=\sum_{F} \int_{\mathscr{R}_{F}} C \boldsymbol{\nu}_{\nu_{F}} \cdot \mathbf{w}_{\mu_{F}}=0 \text {. }
$$

The first of (9.8) and (9.16)-(9.18) yield (9.15).

To establish the energy balance (9.11) we use (2.8), (8.5), (9.1), (9.4), and (9.14) to show that

$$
\left\{\int_{R} \varepsilon\right\}^{\cdot}=-\int_{r}[\mathbf{q}] \cdot \mathbf{m}-\int_{R_{a}} \operatorname{div} \mathbf{q}-\int_{R_{\beta}} \operatorname{div} \mathbf{q}=-\int_{\partial R} \mathbf{q} \cdot \mathbf{n} .
$$

To verify the dissipation inequality (9.12), note first that, by (8.5) and (9.3),

$$
\dot{\varphi}=-\operatorname{div}(u \mathbf{q})+\mathbf{q} \cdot \nabla u
$$

away from the interface, and this relation, (2.8), (9.3), and (9.14) yield

$$
\begin{aligned}
\left\{\int_{R} \varphi\right\}^{\cdot} & =-\int_{,} \frac{1}{2}[c] u^{2} V-\int_{R_{u}} \operatorname{div}(u \mathbf{q})-\int_{R_{\beta}} \operatorname{div}(u \mathbf{q})+\int_{R} \mathbf{q} \cdot \nabla u \\
& =\int_{\Gamma}\left(u[\mathbf{q}] \cdot \mathbf{m}-\frac{1}{2}[c] u^{2} V\right)-\int_{\partial R} u \mathbf{q} \cdot \mathbf{n}+\int_{R} \mathbf{q} \cdot \nabla u .
\end{aligned}
$$

But, by (9.3), (9.4), (9.9), and (9.15),

$$
\begin{aligned}
\int_{\Gamma}\left(u[\mathbf{q}] \cdot \mathbf{m}-\frac{1}{2}[c] u^{2} V\right) & =-\int_{\nu}(\pi+b V) V \\
& =-\left\{\int_{\mu} f\right\}^{\cdot}-\int_{\nu} b V^{2}+\int_{\partial \nu} \mathbf{C} \boldsymbol{\nu} \cdot \mathbf{w}_{\mu} ;
\end{aligned}
$$

(9.21) and (9.22) yield the dissipation inequality (9.12).

Thus the solution of the modified quasi-linear system is consistent with the "first two laws" in the form (9.11) and (9.12).

$b$. Growth theorems. We restrict our attention to a bounded body and to the following types of boundary conditions: an insulated boundary for which

$$
\mathbf{q} \cdot \mathbf{n}=0 \text { on } \partial \Omega \text { for all time; }
$$


an isothermal boundary for which

$$
u=U \text { on } \partial \Omega \text { for all time }
$$

with $U \equiv$ constant the prescribed boundary temperature.

The energy balance and dissipation inequality yield important Lyapunov relations for boundary conditions of this type. Indeed, if in (9.11) and (9.12) we let $R=\Omega$, so that $r=\mathscr{S}$ and $\partial r=\varnothing$, then we find that: ${ }^{18}$ Given a solution of the modified quasi-linear system, if the boundary is insulated,

$$
\left\{\int_{\Omega} \varepsilon\right\}^{\cdot}=0, \quad\left\{\int_{\Omega} \varphi+\int_{\mathscr{S}} f\right\}^{\cdot}=-\mathscr{D}(u, \Omega) \leq 0 ;
$$

if the boundary is isothermal,

$$
\left\{\int_{\Omega}(\varphi-U \varepsilon)+\int_{\mathscr{S}} f\right\}^{\cdot}=-\mathscr{D}(u, \Omega) \leq \dot{0}
$$

(cf. (9.1), (9.2), (9.7), and (9.13)).

By (9.6), these results are valid also for the quasi-linear system provided the specific heat is the same for both phases.

c. Quasi-static approximation. A (common) quasi-static approximation ${ }^{19}$ to both the quasi-linear and modified quasi-linear systems is obtained by setting the specific heats equal to zero:

$$
c_{\alpha}=c_{\beta}=0,
$$

in which case we have the energy balance

$$
\ell\left\{\text { volume }\left(R_{\beta}\right)\right\}^{\cdot}=-\int_{\partial R} \mathbf{q} \cdot \mathbf{n}
$$

and the dissipation inequality

$$
\left\{\int_{r} f\right\}^{\cdot} \leq-\int_{\partial R} u \mathbf{q} \cdot \mathbf{n}+\int_{\partial r} \mathbf{C} \boldsymbol{\nu} \cdot \mathbf{w},
$$

for each control volume $R$, with the difference between the right and left sides of (9.29) given by (9.13). Thus, in particular,

$$
\left\{\text { volume }\left(\Omega_{c}\right)\right\}^{\cdot}=0, \quad\left\{\int_{\mathscr{S}} f\right\}^{\cdot}=-\mathscr{D}(u, \Omega) \leq 0
$$

for an insulated boundary, and

$$
\left\{\ell U \text { volume }\left(\Omega_{\kappa}\right)+\int_{\mathscr{P}} f\right\}^{\cdot}=-\mathscr{D}(u, \Omega) \leq 0
$$

for an isothermal boundary.

d. Justification of the modified quasi-linear system. The quasi-linear system was developed by formally linearizing the PDEs and interface conditions of the general theory. A problem with this procedure is that the thermodynamical structure is lost

\footnotetext{
${ }^{18}$ Cf. Gurtin [1986], §10; [1988], Eqs. (7.8), (7.9).

${ }^{19}$ Rybka [1992] establishes local existence for the corresponding isotropic problem in $\mathbb{R}^{2}$ for a bounded container with walls held at $u=0$.
} 
in the approximation. An alternative procedure-and one that ensures a consistent thermodynamical structure-begins with approximate versions of the thermodynamical laws and generates a theory that is exact within this framework. ${ }^{20}$

We begin with a formal argument in support of (9.11) and (9.12) as the appropriate thermodynamical laws for the modified theory. The condition $(8.2)_{1}$, which represents the interfacial energy balance for the quasi-linear theory, involves no terms representing surface structure, and it seems reasonable to base the modified theory on an energy balance in which such terms are neglected; such a balance is furnished by (9.11). Deducing an appropriate dissipation inequality is more delicate. Roughly speaking, such an inequality should be quadratic in $u$; thus, by (6.10), it seems reasonable to base the theory on (5.19) with $\bar{\varphi}$ and $\bar{u}$ replaced by $\varphi$ and $u$. Also, since $h$ is not present in the energy balance (9.11), we omit it in (5.19). Finally, $e-\vartheta_{T} s=\hat{f}\left(\vartheta_{T}, \mathbf{m}\right)=\hat{f}(\mathbf{m})$ plus higher-order terms, and we therefore replace $e-\vartheta_{T} s$ by $\hat{f}(\mathbf{m})$ in (5.19). This discussion leads us to consider (9.12) as an appropriate version of the dissipation inequality for the modified theory.

We therefore take (9.11) and (9.12) as the basic thermodynamical laws, which we consider in conjunction with the force balance (4.1). Localizing (9.11) and (9.12) with the aid of (9.3) leads to the bulk relations

$$
\dot{\varepsilon}=-\operatorname{div} \mathbf{q}, \quad \dot{\psi}+\varepsilon \dot{u}+\mathbf{q} \cdot \nabla u \leq 0
$$

and to interface conditions consisting of the first of (9.4), the conclusion $f=\sigma$, and

$$
\stackrel{\circ}{f}+(\pi-[\psi]) V \leq 0 .
$$

Writing bulk constitutive equations for each phase in which $\psi, \varepsilon$, and $\mathbf{q}$ depend on $u$ and $\nabla u$ with $\varepsilon$ and $\mathbf{q}$ linear, and demanding consistency with the second of (9.32), leads to the relations

$$
\begin{array}{llll}
\psi=-\frac{1}{2} c_{\alpha} u^{2}, & \varepsilon=c_{\alpha} u, & \mathbf{q}=-\mathbf{K}_{\alpha} \nabla u & \text { in phase } \alpha, \\
\psi=-\ell u-\frac{1}{2} c_{\beta} u^{2}, & \varepsilon=\ell+c_{\beta} u, & \mathbf{q}=-\mathbf{K}_{\beta} \nabla u & \text { in phase } \beta,
\end{array}
$$

with all moduli constant. Interfacial constitutive equations with $f$ and $\pi$ functions of $u$ and $V$, with $\pi$ linear, lead, via (9.33), to the restricted relations

$$
f=\hat{f}(\mathbf{m}), \quad \pi=[\psi]-\hat{b}(\mathbf{m}) V .
$$

Finally, (9.34) yields (9.3) for the Gibbs function $\varphi$. Thus, the modified quasi-linear theory, as described in Sec. 9a, follows as an exact theory based on the approximate thermodynamical laws (9.11) and (9.12) in conjunction with the constitutive equations described above.

e. Perfect conductors. ${ }^{21}$ Consider the quasi-linear-or modified quasi-linearsystem for a bounded region with boundary held at the constant temperature $U$. We now discuss the asymptotic form these equations take when the conductivity of each phase is large. Precisely, we replace

$$
\mathbf{K}_{\mu} \text { by } \delta^{-1} \mathbf{K}_{\mu}, \quad \mu=\alpha, \beta,
$$

\footnotetext{
${ }^{20}$ Gurtin [1993c].

21 Gurtin [1988], §8.
} 
under the assumption that $\delta$ is small. Writing a formal perturbation for $u$ in powers of $\delta$, it is clear that the lowest-order term, also written $u$, should be consistent with

$$
\begin{aligned}
& \operatorname{div} \mathbf{q}=0, \quad \mathbf{q}=-\mathbf{K}_{\mu} \nabla u \quad \text { in bulk, } \\
& {[\mathbf{q}] \cdot \mathbf{m}=0 \quad \text { on the interface }}
\end{aligned}
$$

in conjunction with the interface condition $(8.2)_{2}$ or $(9.4)_{2}$. Under reasonable assumptions the problem (9.37) has the unique solution $u(\mathbf{x}, t) \equiv U$; the only equation then left to solve is the interface condition ${ }^{22}$

$$
b^{F} V_{F}(t)=A_{F}(t)^{-1} \sum_{G} \lambda_{F G} L_{F G}(t)-D
$$

for each facet $F$, where $b^{F}=\hat{b}\left(\mathbf{m}_{F}\right)$, and where $D=\ell U$ for the quasi-linear equations, and $D=\ell U+\frac{1}{2}[c] U^{2}$ for the modified quasi-linear equations.

Acknowledgment. This work was supported by the Army Research Office, by the National Science Foundation, and by the Technical Superior Institute of Lisbon.

\section{REFERENCES}

[1951] C. Herring, Surface tension as a motivation for sintering, The Physics of Powder Metallurgy (W. E. Kingston, ed.), McGraw-Hill, New York

[1963] B. D. Coleman and W. Noll, The thermodynamics of elastic materials with heat conduction and viscosity, Arch. Rational Mech. Anal. 13, 167-178

[1963] F. C. Frank, The geometrical thermodynamics of surfaces, Metal Surfaces: Structure, Energetics, and Kinetics, Amer. Soc. Metals, Metals Park, Ohio

[1972] D. W. Hoffman and J. W. Cahn, A vector thermodynamics for anisotropic surfaces - 1. Fundamentals and applications to plane surface junctions, Surface Sci. 31, 368-388

[1974] J. W. Cahn and D. W. Hoffman, A vector thermodynamics for anisotropic surfaces - 2. Curved and faceted surfaces, Act. Metall. 22, 1205-1214

[1986] M. E. Gurtin, On the two-phase Stefan problem with interfacial energy and entropy, Arch. Rational Mech. Anal. 96, 199-241

[1988] Ben Amar and Y. Pomeau, Growth of faceted needle crystals: Theory, Europhys. Lett. 6, 609-614

[1988] M. E. Gurtin, Multiphase thermomechanics with interfacial structure. 1. Heat conduction and the capillarity balance law, Arch. Rational. Mech. Anal. 104, 195-221

[1988] J. E. Taylor, Constructions and conjectures in crystalline nondifferential geometry (Proc. Conf. Diff. Geom.), Rio de Janeiro, Pittman, London, 1988

[1989] S. Angenent and M. E. Gurtin, Multiphase thermomechanics with interfacial structure. 2. Evolution of an isothermal interface, Arch. Rational Mech. Anal. 108, 323-391

[1990] M. E. Gurtin and J. Matias, Notes of lectures given by Gurtin at the IMA, University of Minnesota, September 1990

[1990] M. E. Gurtin and A. Struthers, Multiphase thermomechanics with interfacial structure. 3. Evolving phase boundaries in the presence of bulk deformation, Arch. Rational Mech. Anal. 112, 97-160

[1991] M. E. Gurtin, On thermomechanical laws for the motion of a phase interface, Zeit. Angew. Math. Phys. 42, 370-388

[1992] F. Almgren and J. Taylor, Flat flow is motion by crystalline curvature for curves with crystalline energies, Rept. GCG43, Geometry Center, U. Minnesota

[1992] J. Taylor, Mean curvature and weighted mean curvature, Act. Metall. 40, 1475-1485

[1992] P. Rybka, A quasi-steady approximation to an integro-differential model of interface motion, preprint

\footnotetext{
${ }^{22}$ Taylor [1988] and Angenent and Gurtin [1989] propose and discuss equations of this type (cf. Fukui and Giga [1992], Taylor [1992], Giga, Gurtin, and Matias [1993], Girão and Kohn [1994], and Girào [1993]).
} 
[1993] T. Fukui and Y. Giga, Motion of a graph by nonsmooth weighted curvature, Proceedings of the First World Congress of Nonlinear Analysts (V. Lakshmikantham, ed.), Walter de Gruyter, Hawthorne, NY (to appear)

[1993] Y. Giga, M. E. Gurtin, and J. Matias, On the dynamics of crystalline motions, forthcoming

[1993] P. M. Girão, Convergence of a crystalline algorithm for the motion of a simple closed curve by weighted curvature, Siam J. Numer. Anal. (to appear)

[1993a] M. E. Gurtin, Thermomechanics of Evolving Phase Boundaries in the Plane, Oxford University Press

[1993b] M. E. Gurtin, Thermodynamics and the supercritical Stefan equations with nucleations, Quart. Appl. Math. 52, 133-155 (1994)

[1993c] M. E. Gurtin and P. W. Voorhees, The continuum mechanics of coherent two-phase elastic solids with mass transport, Proc. Roy. Soc. Lond. A 440, 323-343

[1994] P. M. Girão and R. V. Kohn, Convergence of a crystalline algorithm for the heat equation in one dimension and for the motion of a graph by weighted curvature, Numer. Math. 67, 41-70 